\title{
ON RADIAL FLOW OF A CONDUCTING GAS IN CROSSED FIELDS*
}

\author{
By ERLING DAHLBERG \\ Royal Institute of Technology and Swedish State Power Board \\ Stockholm, Sweden
}

\begin{abstract}
An earlier treatment of quasi-onedimensional flow of a conducting gas in crossed fields (Q.A.M., 19, 177 (1961)) is extended to radial flow with azimuthal magnetic field. The usual simplifying assumptions are made, including constant specific heats; and different flow situations are illustrated by means of phase diagrams (drawn for $\gamma=1.33)$. One of the differences between radial flow $(E=$ const.) and that in exponentially diverging channels $(E / B=$ const.) is the possibility of a smooth transition from supersonic to subsonic motion that seems to exist for a certain range of strongly divergent radial channels.
\end{abstract}

In connection with the interest in magnetohydro-dynamic power generators and propulsion devices, many investigations have lately been made of the quasi-one-dimensional steady flow of a conducting gas in crossed fields (for references, see [1]). Owing to the complexity of the problem, simplifying restrictions have invariably been imposed. We have recently published [1] a qualitative survey of the various types of flow that are possible in a channel with exponentially varying cross section and constant $E / B$ and with wall drag taken into account. Phase diagrams were used to illustrate the variation along the channel of two chosen gas variables, $u^{*} \propto u$ and $p^{*} \propto p S$ (where $u, p$ and $S$ are the gas velocity, pressure and cross-section area). The applicability of this method derives from the possibility of characterizing the flow by two equations of the form

$$
\begin{aligned}
& f(x)\left(d u^{*} / d x\right)=g\left(u^{*}, p^{*}\right), \\
& f(x)\left(d p^{*} / d x\right)=h\left(u^{*}, p^{*}\right),
\end{aligned}
$$

where $x$ denotes distance along the channel and $f(x) \neq 0$ need not be known in detail for a qualitative study. In this note we shall use the same method and essentially the same variables to survey the possible types of flow in another configuration, which has been studied numerically by Podolsky and Borman [2] for a certain range of flow parameters. We consider a wedge-like structure consisting of two parallel, plane electrodes $(E=$ const.) with plane, radially converging or diverging, insulating sidewalls perpendicular to the magnetic field $(B \propto 1 / S)$, Fig. 1 .

We make the usual assumptions: $a$ ) steady flow, $b$ ) scalar conductivity, $c$ ) constant specific heats, $d$ ) negligible heat flow, viscosity and boundary effects except possibly a small wall drag, which may be taken into account by employing the conventional gasdynamic drag coefficient for onedimensional flow, etc. We also assume that the magnetic field due to the currents in the gas is negligible. We choose positive directions as in Fig. 1, and proceed to derive two flow equations of the form (1). This could easily be done directly, but we prefer to start from the basic equations of the earlier work (Ref. [1],

•Received January 29, 1962. 


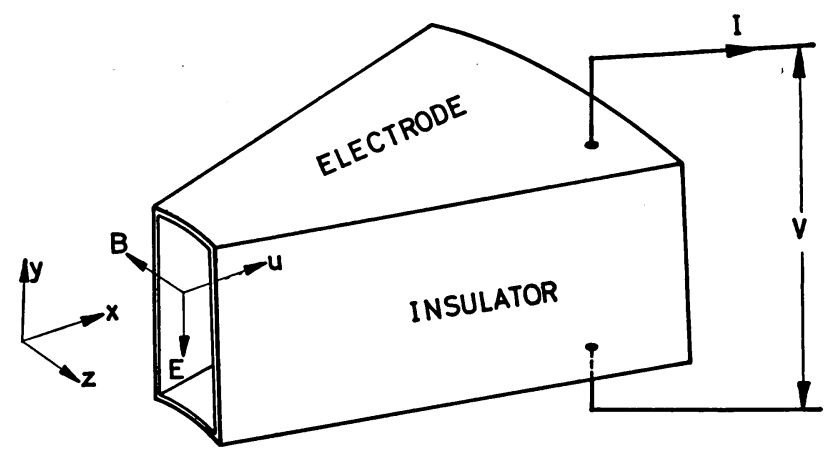

Fig. 1. Outline of flow structure. The conducting gas flows in the channel between the two electrodes and the two, insulating, radial walls. The gas velocity, $u$, and the electric field between the electrodes, $E$, are perpendicular to each other and to the magnetic field, $B$. If $E / B<u$, an induced current, $I$, will flow through the external load. $E / B>u$ requires a supply voltage and gives a current in the opposite direction producing an accelerating volume force, $i B$, on the gas.

Eqs. (2), (3)), namely.

where

$$
\begin{aligned}
& a \frac{d u^{*}}{d x}=\frac{\left(u^{*}-b / b_{0}\right)\left(u^{*}-b / b_{0} \beta\right)+u^{*}\left(\varphi u^{*}-\psi p^{*}\right)}{p^{*}-(\beta-1) u^{*}} \\
& a \frac{d p^{*}}{d x}=-\frac{\left(u^{*}-b / b_{0}\right)\left(p^{*}+u^{*}-b / b_{0}\right)+\left(p^{*}+u^{*}\right)\left(\varphi u^{*}-\psi p^{*}\right)}{p^{*}-(\beta-1) u^{*}},
\end{aligned}
$$

$$
\begin{array}{rlrl}
a & =\rho u / B^{2} \sigma \beta, & u^{*} & =u / b_{0}, \\
b & =E / B, & p^{*}=p \beta / \rho u b_{0}, \\
\psi & =(a / S)(d S / d x), & \varphi & =\beta a 2 f / D, \\
\beta & =c_{p} / R=\gamma /(\gamma-1), & &
\end{array}
$$

and the subscript 0 indicates chosen constant values.

We also introduce

$$
u^{0}=u^{*} b_{0} / b=u B / E, \quad p^{0}=p^{*} b_{0} / b=p \beta B / \rho u E .
$$

MKSA-units are used and the nomenclature is the same as in [1]:

$$
\begin{array}{ll}
u=\text { velocity, } & \sigma=\text { electric conductivity } \\
B=\text { magnetic field, } & \beta=\text { normalized specific heat, } \\
S=\text { cross-section area, } & f=\text { at constant pressure } \\
E=\text { electric field, } & D=\text { hydraulic diameter } \\
p=\text { pressure }, & b=\text { characteristic velocity } \\
\rho=\text { density }, & \varphi=\text { wall drag parameter }
\end{array}
$$

From the definitions of $u_{0}$ and $p_{0}$, we have

$$
\begin{aligned}
& a\left(d u^{0} / d x\right)=\left(b_{0} / b\right) a\left(d u^{*} / d x\right)-(a / b)(d b / d x) u^{0}, \\
& a\left(d p^{0} / d x\right)=\left(b_{0} / b\right) a\left(d p^{*} / d x\right)-(a / b)(d b / d x) p^{0} .
\end{aligned}
$$

For $E / B=b=b_{0}$, i.e. the case studied in [1], $u^{0}$ and $p^{0}$ are equivalent to $u^{*}$ and $p^{*}$. We note that for $E=$ const., $B \propto 1 / S, d S / d x=$ const. (radial flow), we have $a \propto S / \sigma$, $b \propto S, \varphi \propto S / D \sigma$ and

$$
(a / b)(d b / d x)=(a / S)(d S / d x)=\psi \propto 1 / \sigma .
$$


In the following we shall neglect the variations of $\varphi$ and $\psi$ along the channel. This is justifiable if the conductivity is nearly constant and $\varphi \ll 1$ (or $\Delta z_{\max } / \Delta y \ll 1$ ). Further $p^{0} \propto p$ and $u^{0} \propto u / S$ for the radial flow considered here. We also note that the first terms on the right hand side of $\left(2^{\prime}\right)$ and $\left(3^{\prime}\right)$ are identical with the right members of (2) and (3), if we there put $b / b_{0}=1$ and substitute $u^{0}$ and $p^{0}$ for $u^{*}$ and $p^{*}$. This means that, on the critical line $\left(p^{0}=(\beta-1) u^{0}\right)$, the general slope $\left(d p^{0} / d u^{0}=-\beta\right)$ and the position of the pseudo-singular points are the same as in [1]. The singular points and the character of the pseudo-singular points will, however, be different. It should also be noticed that the temperature and enthalpy are not now functions of $p^{0}$ and $u^{0}$ alone.

The new flow equations, valid for the configuration of Fig. 1, are

$$
\begin{aligned}
& a \frac{d u^{0}}{d x}=\frac{\left(u^{0}-1\right)\left(u^{0}-1 / \beta\right)+u^{0}\left[u^{0}(\varphi+(\beta-1) \psi)-p^{0} 2 \psi\right]}{p^{0}-(\beta-1) u^{0}}, \\
& a \frac{d p^{0}}{d x}=-\frac{\left(u^{0}-1\right)\left(p^{0}+u^{0}-1\right)+u^{0}\left[u^{0} \varphi-p^{0}(\beta \psi-\varphi)\right]}{p^{0}-(\beta-1) u^{0}},
\end{aligned}
$$

and the electric power yield per unit length is $i E S=\sigma E^{2} S\left(u^{0}-1\right)=\rho u S\left(u^{0}-1\right) b^{2} / a \beta$.

The different types of phase diagram in the $p^{0} u^{0}$-plane that obtain for various values of $\psi$ and small $\varphi$ have been collected in Fig. 2 (drawn for $\beta=4$ corresponding to $\gamma=1.33$ )
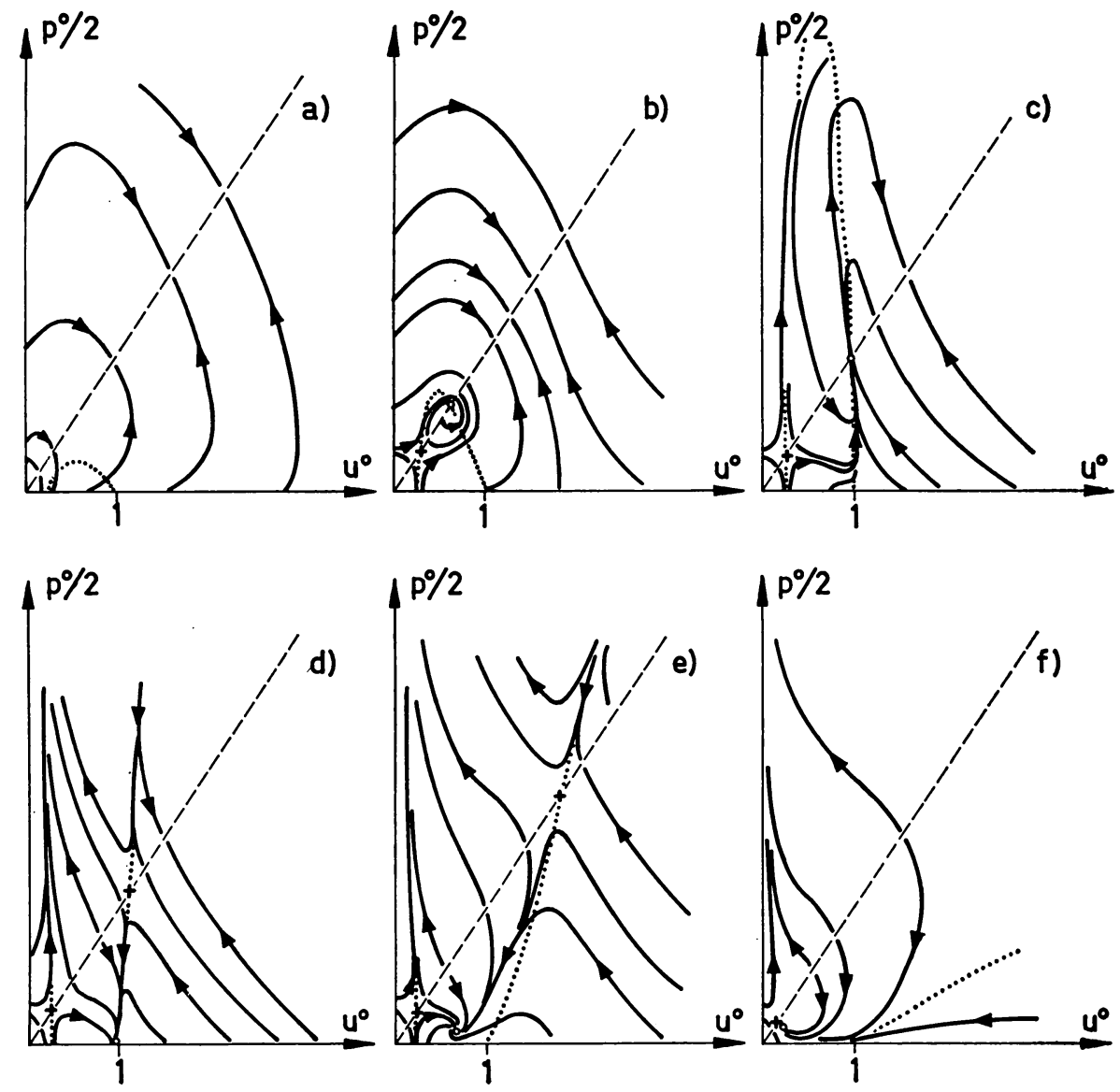

(Continued on next page) 

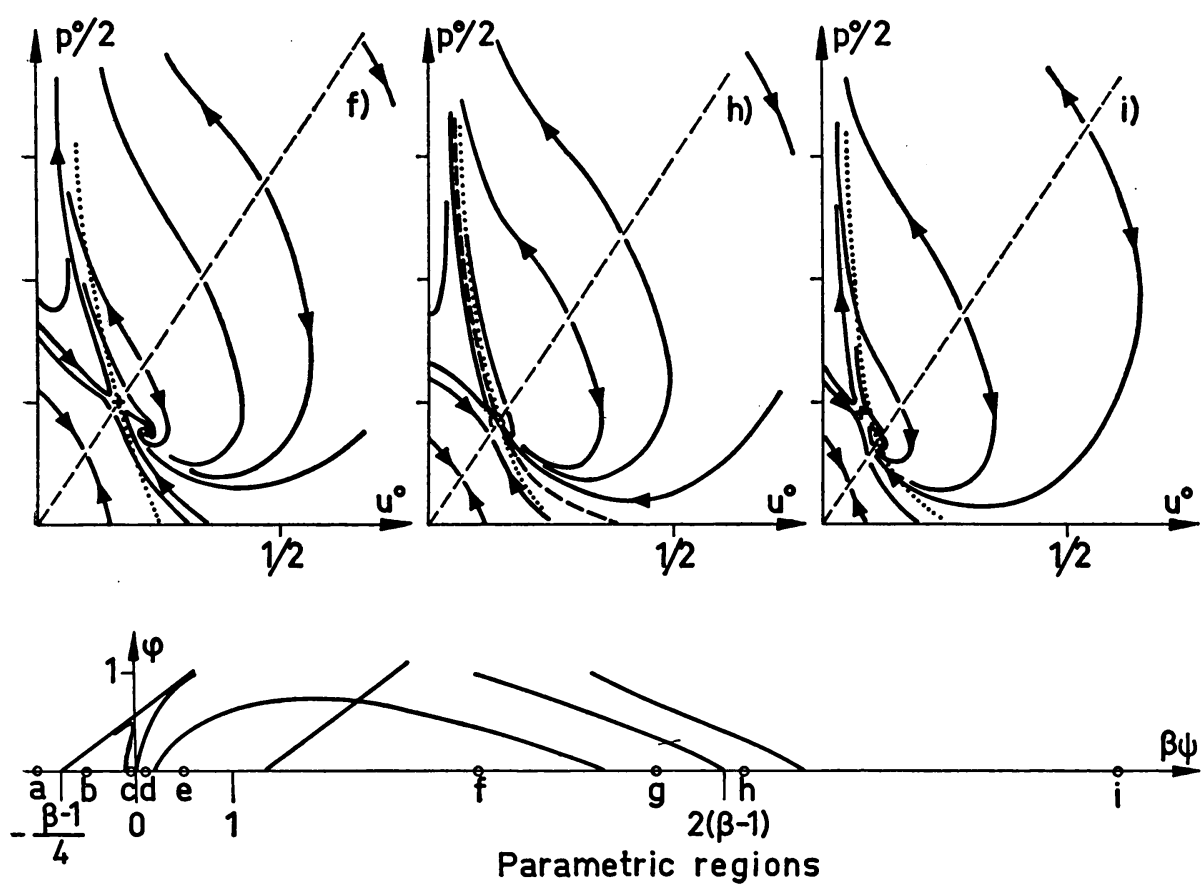

FIG. 2. Phase diagrams. A phase diagram shows the variation along the channel of the normalized velocity, $u^{0} \propto u / S$ (where $S$ is the cross-section area), and the normalized pressure, $p^{0} \propto p$, for given values of the friction and channel divergence parameters, $\varphi$ and $\psi$. The dashed diagonal (critical line) corresponds to sonic velocity. The values of $\varphi(=0)$ and $\psi$ for the different diagrams are indicated by the corresponding letters in the map of parametric regions. In $d, e, f$ (and $g$ ) we note the existence of a stable equilibrium point in the supersonic range, corresponding to a flow with constant pressure and linearly increasing velocity. We also note the smooth transition from supersonic to subsonic motion that is characteristic for the regions $c$ and $h$.

together with a sketch indicating what regions in the $\varphi \psi$-plane correspond to the various types of phase diagram illustrated. (The region $g$ has not been illustrated; it differs from $f$ only in that the singular point is nodal instead of focal and lies even closer to the critical line on the supersonic side.) We note that the singular point $\left(u^{0}=1 /(1+\varphi+\beta \psi)\right)$ lies outside the physically relevent part of the phase plane for $\psi<0$; in regions $d$ and $g$ it is a stable nodal point in the supersonic range, in regions $e$ and $f$ it is a supersonic stable focal point, in regions $h$, and $i$ (as well as $c$ for $\psi>0$ ) it is a saddle point in the subsonic range. Regarding the pseudo-singular points on the sonic line $\left(p^{0}=(\beta-1) u^{0}\right)$, (cf. [1] and Fig. 3), we find that no such points exist in region $a$, and that when they exist they are two in number. The one nearest the origin is always in the first quadrant $\left(0<u^{0}<2 /(\beta+1)\right)$; it is a "saddle point" in regions $b, c, d, e, f$, and $g$; however, in region $h$ it is a "nodal point, stable from below", and in region $i$ "focal and stable from below". This fact constitutes one of the main differences between radial flow and the flow studied in [1]. The pseudo-singular point farthest from the origin is "focal" in $b$ and "nodal and stable from below" in $c$ (in both regions $2 /(\beta+1)<u^{0}<1$ ), in regions $d$ and $e$ it is a "saddle point". (As in the earlier case there is an extremely narrow region between $a$ and $b$ where it is "nodal and stable from 


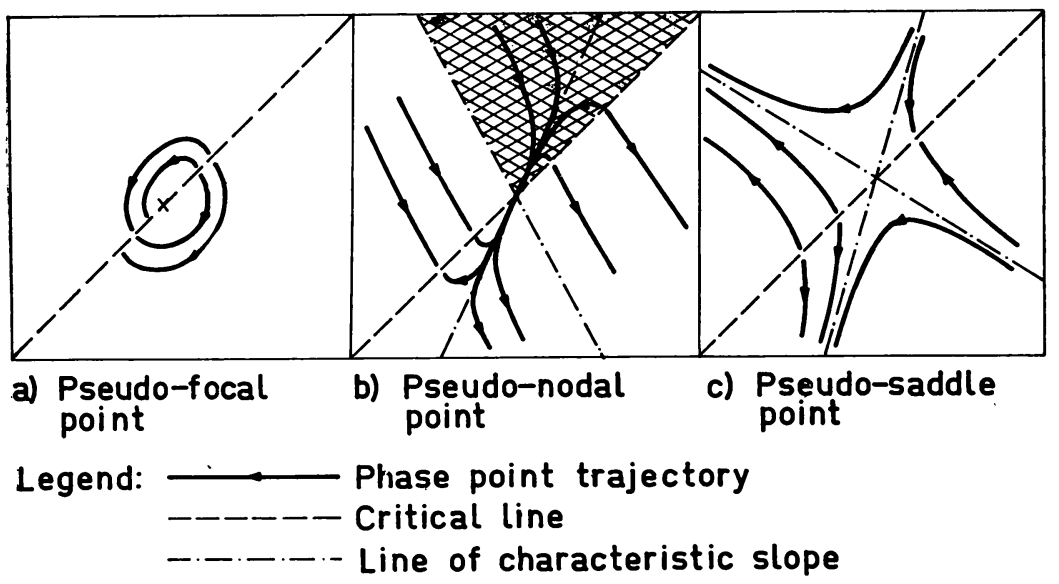

Fig. 3. Pseudo-singular points.

above".) In the regions $f, g, h$, and $i$ it falls in the third quadrant $(E / B<0)$ and is normally "focal".

Apart from the appearance of a pseudo-nodal point for large divergence parameters, we note the following difference between radial flow and that studied before. Whereas a stable singular point in the phase diagrams of [1] represented constant velocity and linearly decreasing pressure, it now represents constant pressure and linearly increasing velocity (cf. Podolsky and Borman's solutions of the first kind, $Q=1 / \beta \psi>(\gamma-1) / 2=$ $\left.\frac{1}{2}(\beta-1)\right)$. Regions of accelerated and decelerated flow are separated in the phase diagrams by the dotted lines as well as by the sonic line.

The pseudo-nodal point found for region $h$ in the $\varphi \psi$-plane deserves further consideration. If the separatrix of the nodal point turns back to the critical line on the subsonic side, any smooth transition occurring will hardly be distinguishable from a weak shock in connexion with a pseudo-focal point (region $i$ ). If, however, the separatrix runs free of the subsonic saddle point, as it seems to do for parameter values near the point $h$, an observable smooth transition might occur. (In phase diagram $h$, the probable path of the separatrix is plotted as a dashed line.)

\section{REFERENCES}

1. E. Dahlberg, On the one-dimensional flow of a conducting gas in crossed fields. Quart. Appl. Math. 19, 177-193 (1961)

2. B. Podolsky and G. Borman, The electromagnetic acceleration of a continuously flowing plasma, pp. 12-29 in S. W. Kash (Ed.), Plasma acceleration, Stanford Univ. Press, 1960 